\title{
Optical label swapping and packet transmission based on ASK/DPSK orthogonal modulation format in IP-over-WDM networks
}

Chi, Nan; Xu, Lin; Christiansen, Lotte Jin; Yvind, Kresten; Zhang, Jianfeng; Holm-Nielsen, Pablo Villanueva; Peucheret, Christophe; Zhang, Jianfeng; Jeppesen, Palle

Published in:

Technical Digest Optical Fiber Communication Conference 2003

Link to article, DOI:

10.1109/OFC.2003.1248576

Publication date:

2003

Document Version

Publisher's PDF, also known as Version of record

Link back to DTU Orbit

Citation $(A P A)$ :

Chi, N., Xu, L., Christiansen, L. J., Yvind, K., Zhang, J., Holm-Nielsen, P. V., Peucheret, C., Zhang, J., \& Jeppesen, P. (2003). Optical label swapping and packet transmission based on ASK/DPSK orthogonal modulation format in IP-over-WDM networks. In Technical Digest Optical Fiber Communication Conference 2003 (Vol. 2, pp. 792-794). IEEE. https://doi.org/10.1109/OFC.2003.1248576

\section{General rights}

Copyright and moral rights for the publications made accessible in the public portal are retained by the authors and/or other copyright owners and it is a condition of accessing publications that users recognise and abide by the legal requirements associated with these rights.

- Users may download and print one copy of any publication from the public portal for the purpose of private study or research.

- You may not further distribute the material or use it for any profit-making activity or commercial gain

- You may freely distribute the URL identifying the publication in the public portal 


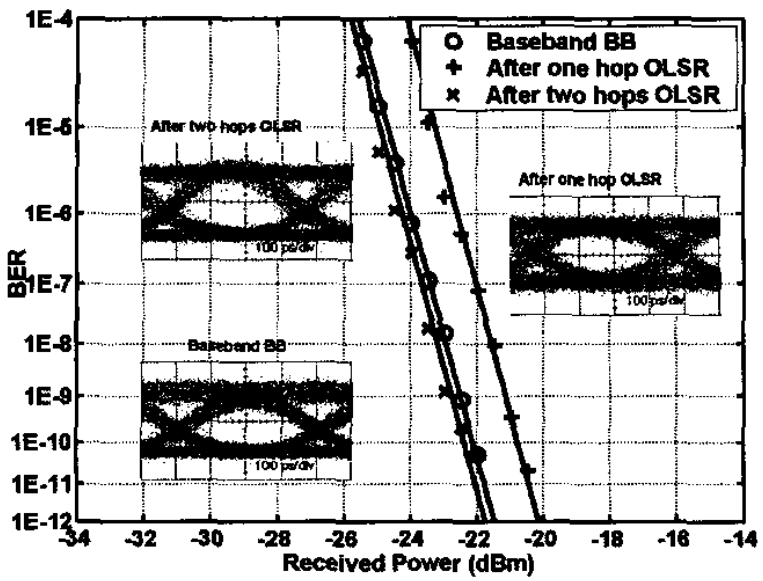

Fig. 3. BER test results of the cascaded OLSR

(Insets: eye diagrams of the baseband payload and signals after OLSR)

and L2' cause the wavelength of the TLD2 to be switched to $\lambda 1^{\prime}(1546 \mathrm{~nm})$ and $\lambda 2^{\prime}(1542 \mathrm{~nm})$ respectively. TLD2 drives the SOA2 that converts the payload signal onto the desired wavelength by cross-gain modulation. P1 converted to $1546 \mathrm{~nm}$ will be routed to the destination port. Fig 2(c) shows $\mathrm{P} 1$ on the destination port. P2 converted to $1542 \mathrm{~nm}$ will be dropped after AWGR. The switched data payload P1 goes to the data receiver for BER measurements. Here the switching with new labels $\mathrm{L} 1^{\prime}$ and $\mathrm{L} 2$ ' emulates the OLSR3 in Fig 1 (a).

Packet by packet bit-error-rate measurements took place on the Pl at each hop. Fig. 3 shows the measured data. Each packet is 600 ns long with a $200 \mathrm{~ns}$ guard time, thus each packet period is $800 \mathrm{~ns}$. The bit pattern was $2^{15}-1$ pseudo-random bit sequence (PRBS) truncated into the packets. The three curves in Fig. 3 are for the optical baseband back-to-back and the payload signals after one and two hop OLSR, respectively. The signal after one hop shows about $0.7 \mathrm{~dB}$ power penalty compared to the baseband payload signal. However, a negative power penalty about $0.2 \mathrm{~dB}$ at BER $=1 \mathrm{e}-9$ appears after 2 hop OLSR, which is mainly due to the $2 \mathrm{R}$ regeneration in the SOAbased MZI WC and the decrease of the received average power after two packet-dropping. The eye diagrams of the switched payload are shown as the insets in Fig. 3. All eye diagrams show clear openings. The signal-to-noise ratio was higher for the second hop compared to the first hop due to the $2 \mathrm{R}$ regeneration of $\mathrm{MZI}$ WC. Also the XGM based SOA wavelength converters invert the logic of the signal which leads to the change of the average power of signal. This results in a higher average power for the first hop. For the second hop the second XGM wavelength converter will invert the logic back to normal. For these reasons, the power received by the date receiver corresponds to different ratios of the real packet power for the 1-hop operation and the 2 hop operation. The combination of the $2 \mathrm{R}$ regeneration and the optical power change leads to the negative power penalty for the 2-hop operation.

\section{Summary}

We have demonstrated for the first time, to our knowledge, the error-free multi-hop cascaded operation of an all-optical label routing system with optical label swapping. The experiment emulated optical packet switching through 2 hops in the network. Experiment results show regenerating optical label switching with label swapping and $2 \mathrm{R}$ packet regeneration. The two-hop routing OLSR system demonstrates negative power penalty of $0.2 \mathrm{~dB}$ at $\mathrm{BER}=1 \mathrm{e}-9$ for data packets.

\section{References}

[1] S.J.B. Yoo, G.K. Chang, High-Throughput, Low-Latency Next Generation Intemet Using Optical Tag Switching, in U.S. Patent 6,111,673. 1997, Bellcore: U.S.A. 1499, August 1999. 635-637 (2001) ECOC2002, (2002). 998665, and by the support of OIDA.

[2] D. J. Blumenthal, A. Carena, L. Rau, V. Curri, and S. Humphries, "All-Optical Label Swapping with Wavelength Conversion for WDM-IP Networks with Subcarrier Multiplexed Addressing," IEEE Photon. Technol. Lett., vol. 11, pp. 1497-

[3] H. J. Lee, S. J. B. Yoo, V. K. Tsui and S. K Fong, "A simple all-optical label detection and swapping technique incorporating a fiber Bragg grating filter," IEEE Photon. Tech. Lett. 13, pp.

[4] Jing Cao, Zhong Pan, Min Yong Jeon, Yash Bansal, Julie Taylor, Venkatesh Akella, Shin Kamei, Katsunari Okamoto, S. J B Yoo "Cascaded Operation of An Optical Packet Routing System with Optical-Label Switching and 2-R Regeneration," Technical Digest, paper \#5.5.5

[5] Min Yong Jeon, Zhong Pan, Jing Cao, and S. J. B. Yoo, "All optical sub-carrier label swapping with $2 \mathrm{R}$ regeneration," submitted to OFC2003. [6] H. J. Lee, V. Hernandez, V. K. Tsui and S, J. B. Yoo, "Simple, polarization-independent, and dispersion-insensitive SCM signal extraction technique for optical switching systems applications," IEE Electron. Lett. 37, pp. 1240-1241 (2001)

[7] S. J. B. Yoo, Hyuek Jae Lee, Yanda Zhang, S K. H. Fong, Vincent Hernandez, Vincent K. Tsui, Srikanth Vaidianathan, Katsunari Okamoto, Shin Kamei, "High-Speed All-Optical Packet Routing System with Optical-Label Switching and Optical-Label Swapping using Rapidly Tunable Wavelength Conversion and a Uniform-Los Cyclic Frequency AWGR," IEEE photonics technology letters, 14, no. 8, pp. 1211-1213 (2002).

This work was partially funded by the Defense Advanced Research Projects Agency (DARPA) and Air Force Research Laboratory under agreement number F30602-00-2-0543, by the National Science Foundation under grant number ANI-

FS2

10:45 AM

Optical Label Swapping and Packet Transmission Based on ASK/DPSK Orthogonal Modulation Format in IP-overWDM Networks

N. Chi, L. Xu, L. Christiansen, K. Yvind, J. Zhang, P. Holm-Nielsen C. Peucheret, C. Zhang, P. Jeppesen, Research Center COM, Technical University of Denmark, Lyngby, Denmark, Email: nc@com.dtu.dk.

We demonstrate all-optical label swapping based on SOA, EAM and HNLF for a two-level optically labeled packet using orthogonal ASK/DPSK modulation format. The ASK/DPSK packet is successfully transmitted over $80 \mathrm{~km}$ NZDSF
1. Introduction

All-optical label switching is of increasing interest in future packet-switched WDM networks because individual packets can be switched through an optical network element without being converted from optical to electronic format [1]. Although the optical wavelength can serve as an optical label in the MP $\lambda S$ scheme [1], a second level of optical label is still necessary for provisioning, maintaining, and restoring switched light-paths. This second level optical label can be realised by sub-carrier modulation [2] or by an orthogonal modulation format combining amplitude shift keying (ASK) and differential phase shift keying (DPSK) modulation on a single carrier [3].

The structure of the optical label switching system for the ASK/DPSK packets is illustrated in Fig, 1. At the edge router the DPSK labels are added to the optical packets without modifying the ASK payload. The intermediate routers perform routing and forwarding operations within the local access networks or metropolitan networks by wavelength conversion and DPSK label swapping. However at the core router, switching is based on larger granularity such as wavelength, therefore only wavelength swapping is required but the DPSK label is preserved.

In this paper we demonstrate all-optical label swapping and packet transmission for a $10 \mathrm{Gbit} / \mathrm{s}$ ASK payload with $2.5 \mathrm{Gbit} / \mathrm{s}$ DPSK label. In the intermediate node, two-level optical label (wavelength label and DPSK label), are swapped using electro-absorption modulators (EAM) or semiconductor optical amplifiers (SOA), and in the core node the wavelength label is swapped while the DPSK label is entirely replicated through the four-wave mixing (FWM) effect in a highly-nonlinear fibre (HNLF). The transmission properties of the ASK/DPSK packet over $80 \mathrm{~km}$ non-zero dispersion shifted fiber (NZDSF) are also investigated. The successful label swapping and transmission experiment clearly demonstrate the feasibility of this combined modulation format scheme.

\section{Optical label processing/swapping}

The experimental setup is shown in Fig. 1(a). The signal source is a wavelength tunable externa cavity laser (TL) working at $1552.5 \mathrm{~nm}$. In the edge router, the optical carrier is first intensity modulated at $10 \mathrm{Gbit} / \mathrm{s}$ by a chirp-free Mach Zehnder modulator. The DPSK label at $2.5 \mathrm{Gbit} / \mathrm{s}$ is then impressed by the subsequent phase modulator, thus making the optical packets ready for transmission. As we have reported before [4], a limited extinction ratio of $3-4 \mathrm{~dB}$ for the payload is used in order to detect the DPSK label.

2.1 Intermediate node function: $\lambda$-label as well as DPSK label swapping

Both the $\lambda$-label and the DPSK label are swapped at the intermediate nodes so that an appropriate optical path can be built-up in the transmission fiber link. The DPSK label can be erased by an intensity-sensitive wavelength converter that copies the payload information onto a new wavelength while omitting the phase information of labels. In our experiment we erase the DPSK label by cross-gain modulation (XGM) -based wavelength conversion in a SOA. A narrow fiber Bragg grating is deployed directly after the SOA to overcome pattern dependence and to remove the frequency chirp [5]. Because the chirp induced by EAM-based wavelength conversion is negligible [6], the phase of the probe and pump signals is not affected in the wavelength conversion process. Therefore the DPSK label can be inserted by phase-modulating a new lightwave and then copying the payload onto it through cross-absorption modulation in an EAM. The SOA and the EAM used in our experiment were kindly provided by GIGA-Intel. At the receiver side, the ASK/DPSK packet is split into two parts after a $3 \mathrm{~dB}$ coupler, so that the ASK payload and the DPSK label can be detected separately. The DPSK label is demodulated using a one bit delay fibre interferometer before direct detection. Fig. 2(a) shows the BER performance and the eye diagrams of the converted payload and the new label. 


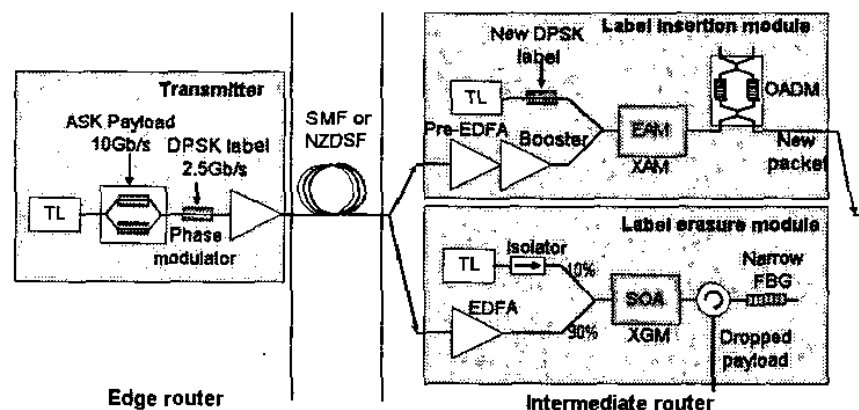

Edge router

\begin{tabular}{|c|c|c|c|c|c|c|}
\hline DPSK label 1 & \multirow{2}{*}{$\begin{array}{l}\text { DPSK Label } \\
\text { processing } \\
\text { and eresure }\end{array}$} & & \multirow{2}{*}{$\begin{array}{l}\text { Wavelength } \\
\text { conversion }\end{array}$} & \multirow{2}{*}{\multicolumn{2}{|c|}{$\begin{array}{c}\text { DPSK } \\
\text { Label } \\
\text { Insertion }\end{array}$}} & DPSK label 2 \\
\hline$\lambda$-label 1 & & $\lambda$-label 1 & & & & $\lambda$-label 2 \\
\hline ASK payload 1 & & ASK poylo & ad 1 & ASK payto & & ASK peyload 1 \\
\hline
\end{tabular}

(b)

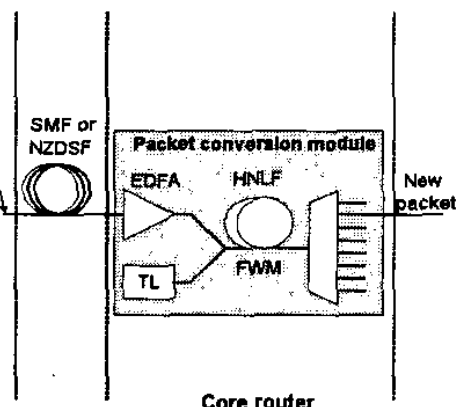

Core router

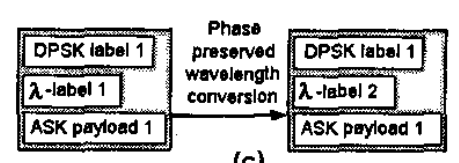

(c)

Fig. 1. (a) Network architecture for optical packet switching based on orthogonal modulation formats and schematics of two-level label swapping in (b) intermediate router and (c) core router.
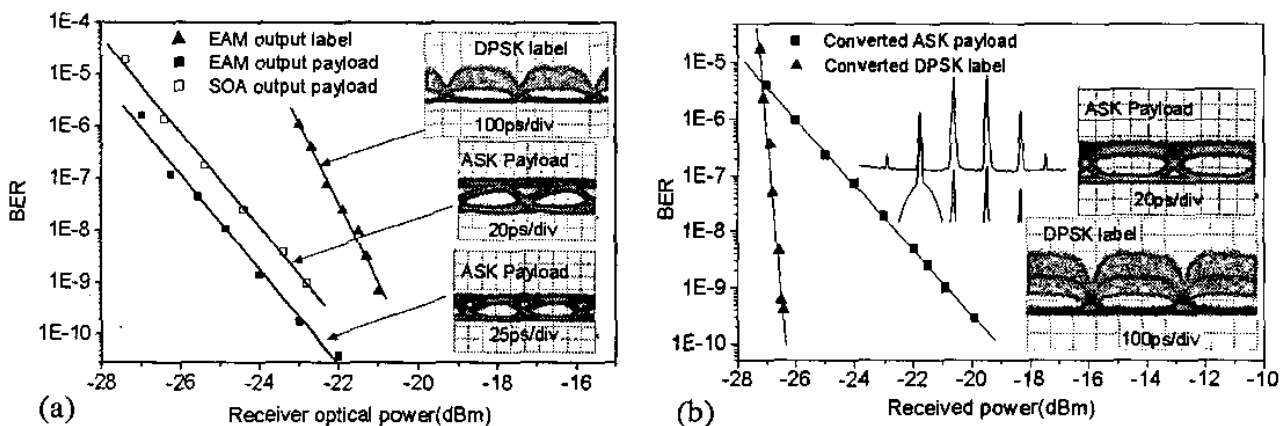

Fig. 2. (a) Measured BER performance and eye diagrams for the converted payload and inserted new DPSK label in the intermediate node based on SOA and EAM, (b) BER performance and the eye diagrams of the wavelength converted payload and the replicated DPSK label in the core node. The inset spectra show the FWM output of the HNLF and the spectrum of the converted signal after AWG,

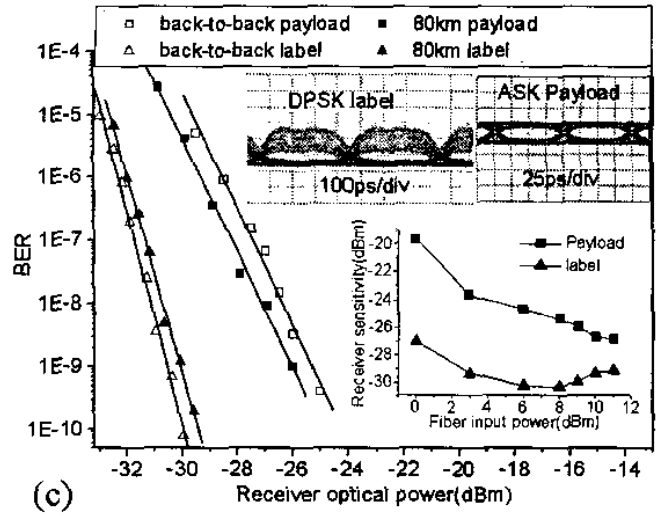

Fig. 2. (c) measured BER performance for back-to-back and after 80km NZDSF transmission; the inset curve shows the receiver sensitivity of the ASK payload and DPSK label as a function of fiber input power.

Very clear and open eyes can be obtained for both the payload and the label after wavelength conversion and label insertion. Because of the influence of the ASK payload, we observe the splitting of the " 0 " level and " 1 ' level for the demodulated DPSK label [4]

2.2 Core node function: $\lambda$-label swapping, DPSK label transparent

In the core routers, a group of wavelength channels are converted to another group, i.e., only $\lambda$ label swapping is performed whereas the DPSK labels are simply replicated. To realize phase-preserving wavelength conversion of this ASK
DPSK modulated signal, interferometric devices based on cross-phase modulation (XPM) in SOAs can not be applied. Therefore some transparent wavelength conversion scheme must be considered to maintain the phase information. Wavelength conversion employing the FWM process in an HNLF is deployed in our core node experiment because FWM wavelength conversion has advantages that include large spectral and dynamic range as well as strict bit rate and modulation format transparency [7].

The FWM process takes place in the $500 \mathrm{~m}$ long HNLF. The zero dispersion wavelength is 1553.6 $\mathrm{nm}$ and the dispersion slope is $0.022 \mathrm{ps} / \mathrm{nm} 2 / \mathrm{km}$.
An arrayed waveguide grating (AWG) with channel spacing of $200 \mathrm{GHz}$ is used to filter out the converted signal. Fig. 2(b) shows the optical spectra of the FWM output from the HNLF and of the converted signal after the AWG. By changing the polarization state of the polarization controller and the input power level, a conversion efficiency of the FWM process of up to $-15 \mathrm{~dB}$ could be achieved. The measured receiver power penalty of the label is $<1.6 \mathrm{~dB}$. However, the power penalty of the payload is about $5 \mathrm{~dB}$ due to the relatively high bit rate and low extinction ratio. The converted label has clearly a multi-level structure which is similar to the back-to-back case.

\section{Transmission properties of ASK/DPSK} packets

The transmission span consists of $80 \mathrm{~km}$ NZDSF and matching dispersion compensating module. $\mathrm{A}$ pre-compensation scheme is adopted due to its better performance than the post-compensation scheme for the ASK payload [4]. Both the payload and the label can be obtained error-free after $80 \mathrm{~km}$ transmission. The DPSK label shows a low power penalty of $0.5 \mathrm{~dB}$. The receiver sensitivity of the payload after transmission is observed to be even enhanced by $0.7 \mathrm{~dB}$ compared to the backto-back case, because the pulses are significantly broadened after the DCF due to the self phase modulation (SPM) but the following NZDSF acts on the chirped pulse to compress them to a width narrower than they were initially. The receiver sensitivities of the payload and the label as a function of the span input power are also measured, as illustrated in Fig. 2(c). When varying the power from 15 to $0 \mathrm{dBm}$, a large input power is found to be the best for the payload whereas an optimum point for the label detection is found to be $8 \mathrm{dBm}$ as shown in the inset of Fig. 2(c). This can be 
explained by the fact that the large input power increases the cross-talk stemming from SPM and as a consequence will influence the DPSK label. Compared to our earlier experimental transmission over $50 \mathrm{~km}$ SMF of the ASK/DPSK packets [8], the usage of NZDSF provides a considerable improvement.

\section{Conclusion}

We have presented our new approach for optical label swapping based on SOA, EAM and HNLF for the two-level optically labelled packets using the ASK/DPSK modulation format, which is performed in the IST STOLAS (Switching Technologies for Optically Labeled Signals) project. The two-level optical label ( $\lambda$-label and DPSK label) can be selectively swapped depending on the network location. Error-free transmission of both payload and label has been demonstrated over an $80 \mathrm{~km}$ NZDSF dispersion compensated span.

\section{References}

$1 \mathrm{~N}$. Ghani, "Lambda-labeling: a framework for IP-over-WDM using MPLS," Optical Networks Magazine, vl, 45-58 (2000).

2 D. J. Blumenthal, et al, "All-optical label swapping with wavelength conversion for WDM-IP networks with subcarrier multiplexed addressing," IEEE Photon. Technol. Lett., v11, 14971499 (1999).

3 T.Koonen, et al, "Optical packet routing in IPover-WDM networks deploying two-level optical labelling," Proc. ECOC'01, (2001).

$4 \mathrm{~N}$. Chi, et al, "Dispersion management for twolevel optically labeled signals in IP-over-WDM networks," ECOC'2002, 5.5.1, (2002).

5 P. Mahgereftech, et al, "Technique for suppression of patterm dependence in a semiconductoroptical-amplifier wavelength converter," IEEE Photon. Technol. Lett., v9, 1583-1585 (1997).

$6 \mathrm{~L}$. Xu, et al "Bandwidth and chirp characterisation of wavelength conversion based on electroabsorption modulators," ECOC2002, P1.26, (2002)

7 T. J. Morgan, et al, "C-band to L-band shift of $80 \mathrm{~nm}(10 \mathrm{THz})$ using four-wave mixing in semiconductor optical amplifier," OFC'99, PD37, (1999)

$8 \mathrm{~N}$. Chi, et al, "Transmission Performance of All-optically labelled Packets using ASK/DPSK Orthogonal Modulation," LEOS'2002, MF3, (2002).

FS3

11:00 AM

Multistage Architectures for Optical Packet Switching Using SOA-Based Broadcast-andSelect Switches

C. Develder, J. Cheyns, M. Pickavet, P. Demeester, Ghent Univ., Gent, Belgium, Email: chris.develder@intec.rug.ac.be.

Optical packet/burst switches can be scaled to high port counts using multi-stage architectures. To reduce the number of switching elements in SOA-based broadcast-and-select architectures, we deploy only a few stages while exploiting the WDM dimension.

\section{Introduction}

To satisfy the ever-lasting bandwidth hunger, (D)WDM is adopted. Long-term strategies for optical networking, that will replace the currently predominant point-to-point systems, envisage Optical Packet Switching (OPS) and/or Optical Burst Switching (OBS), fully exploiting the potential of advanced optical switching technologies. The European research project DAVID [1] aims at proposing viable approaches towards OPS. It uses a broadcast-and-select switch based on SOAs [2]

This paper illustrates how OPS/OBS switches can be scaled to high port counts using a multistage architecture. Section 2 introduces the two most wide-spread node architectures for OPS/OBS and highlights the factors limiting scalability. In Section 3 we review the three-stage Clos architecture, and show how to eliminate a switching stage by using the WDM dimension through wavelength converters. In Section 4 we present a case study for the DAVID architecture showing the advantage of the two-stage architecture in terms of number of switching elements (SOA gates). Section 5 concludes the paper.

\section{Node architectures for OPS/OBS}

The core functionality of an optical packet switch is to selectively transmit packets from a particular input port to a particular output port. "Port" implies a certain wavelength on a certain fibre. For "packet" switching, two fundamentally different approaches exist: one can either opt for fixed length, or rather variable length packets. The network can be operated in a time-slotted manner or an asynchronous mode. Usually a slotted approach is taken for fixed length packets, whereas asynchronous operation is adopted for variable length packets. We reserve the term OPS for fixed-length packet switching using a slotted operation, whereas OBS clearly is a case of unsynchronized switching of variable length packets.

\subsection{Switch fabric architectures}

An optical packet switch from a generic viewpoint comprises three parts [3]: an input interface, a switching fabric, and an output interface. Two architectures dominate the OPS/OBS approaches proposed in recent publications and research projects: (i) broadcast-and-select (B\&S) architectures, (ii) Arrayed Waveguide Grating (AWG) architectures.

B\&S has been proposed in e.g. the European research projects KEOPS and DAVID. The switching fabric proposed by the latter is depicted in Figure 1 [2]. The first stage multiplexes different wavelengths into a single fibre, and jointly amplifies them to compensate for the subsequent power splitting stage. For each output wavelength, two switching stages are foreseen: the first selects one of the F input fibres, and the second selects a single wavelength among the $W$ available ones. Advantages of $B \& S$ include that it is non-blocking, and that it can perform multicasting.

The optical switching technique based on an AWG is adopted by e.g. the WASPNET project, and the more recent STOLAS [4]. The wavelength of a signal offered at one of the AWG's input ports determines via what output port it will leave: with Tuneable Wavelength Converters (TWCs) at its inputs, an AWG can be used as a switching fabric. To construct a fabric for $F$ fibres, each carrying $\mathrm{W}$ wavelengths, in principle the tuneable wavelength converters have to range only over the $\mathrm{W}$ wavelengths in use. Unfortunately, the resulting switch then is a blocking one: there is no guarantee that all packets can be forwarded to a certain output, even if we have only to switch W (or less) packets to each of the output fibres. However, a non-blocking fabric is obtained with converters tuneable over $\mathrm{F} \times \mathrm{W}$ wavelengths. In this case, additional converters (fixed output wavelength) at the outputs are needed.

\subsection{Scalability}

Both the B\&S and AWG architectures have limited scalability. The factor limiting the port coun for the $\mathrm{B} \& \mathrm{~S}$ architecture is the splitting ratio: each incoming signal is split in the broadcast stage over each of the $\mathrm{N}=\mathrm{F} \times \mathrm{W}$ output ports. For the AWG-based approach, the number of output ports is limited by the tuneability range of the TWCs, which have to be tuneable over as many wavelengths as there are output ports $(\mathrm{N}=\mathrm{F} \times \mathrm{W})$ A possible solution is the adoption of multistage architectures, as discussed in the next section.

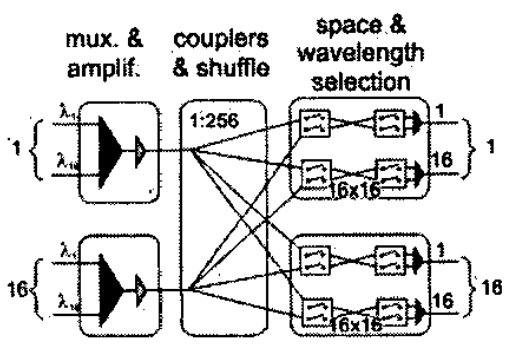

Fig. 1. The DAVID broadcast-and-select architecture.

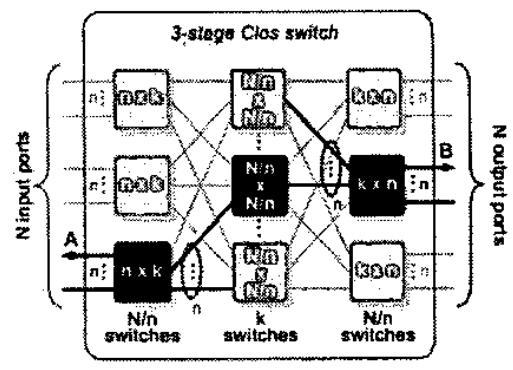

Fig. 2. Three-stage Clos architecture.

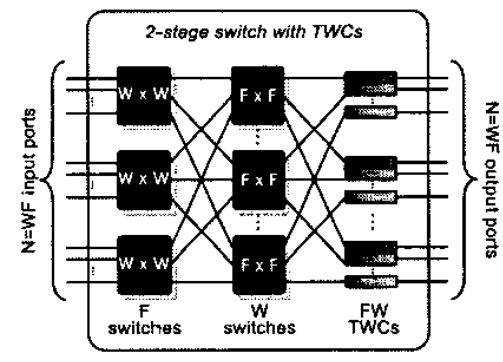

Fig. 3. Two-stage architecture with TWCs.

\section{Multistage architectures}

In the early days of network engineering, Clos proposed a multistage architecture for large switches based on smaller building blocks [5]. A sketch of an $\mathrm{N} \times \mathrm{N}$ switching architecture using a three-stage Clos network is outlined in Figure 2. The three stages comprise (i) $\mathrm{N} / \mathrm{n}$ switches of dimension $n \times k$, (ii) $k$ matrices $(N / n) \times(N / n)$, and (iii) $N / n$ switches of size $k \times n$

To be non-blocking, a lower bound on $k$ is imposed: $k \geq 2 n-1$. This minimal value can easily be determined as follows: consider a connection between input ports $\mathrm{A}$ and $\mathrm{B}$. The worst case occurs when (i) all n-1 other ports of the firststage to which A belongs are already occupied and connected to some output ports via n-l second stage switches; and (ii) the (n-1) remaining ports of the third-stage switch with $B$ also are occupied, coming from $n-1$ other intermediate stage switches. Therefore the switch needs at leas $(n-1)+(n-1)+1$ connection points, hence $k \geq 2 n-1$. Typically, it is convenient to set $k=2 n$. This boundary was determined by Clos for circuitswitched networks, implicitly assuming that connections cannot be rerouted along other (second stage) switches once they have been set-up.

Unlike Clos, we focus on OPS, operating in slotted mode. In this case, the bound for $k$ is that of a rearrangeable non-blocking switch, which is considerably smaller: $k=n$. The proof why $k=n$ suffices is a well-known result from graph theory (the problem is equivalent to a graph coloring problem in a bipartite graph with degree $n$, which is $\mathrm{n}$-colorable)

In case of OPS, all building block switch fabrics have symmetrical dimensions. If all wavelengths within a fibre may be considered equivalent, $N$ 